\title{
Electrically tunable metasurface with independent frequency and amplitude modulations
}

Jin Zhang, Xingzhan Wei, Ivan D. Rukhlenko, Hou-Tong Chen, and Weiren Zhu

\section{Supplementary Information}

Fig. S1 shows the simulated coefficient of the proposed metasurface with different capacities and Fermi levels for y polarization. Compared to the results for $x$ polarization shown in Fig. 1, similar tunability of amplitude and frequency can be found for $y$ polarization. Specifically speaking, increasing the capacitance of the varactor diodes from $0.16 \mathrm{pF}$ to $1.1 \mathrm{pF}$ shifts the working frequency from $3.25 \mathrm{GHz}$ to $1.9 \mathrm{GHz}$. Meanwhile, the amplitude modulation also can be realized from less than $-30 \mathrm{~dB}$ to nearly $-10 \mathrm{~dB}$ by tuning the Fermi level of graphene from 0 to $0.12 \mathrm{eV}$. Due to the asymmetry of the metal structure, the resonant frequency for $y$ polarization experiences a red shift compared to the one with $x$ polarization. However, the sample of our fabricated metasurface is not large enough to be measured in WR430 waveguide system (size: $109 \mathrm{~mm} * 55 \mathrm{~cm}$; frequency:1.72 2.6GHz) because of the limitation of synthesis and transform high-quality graphene in large scale.

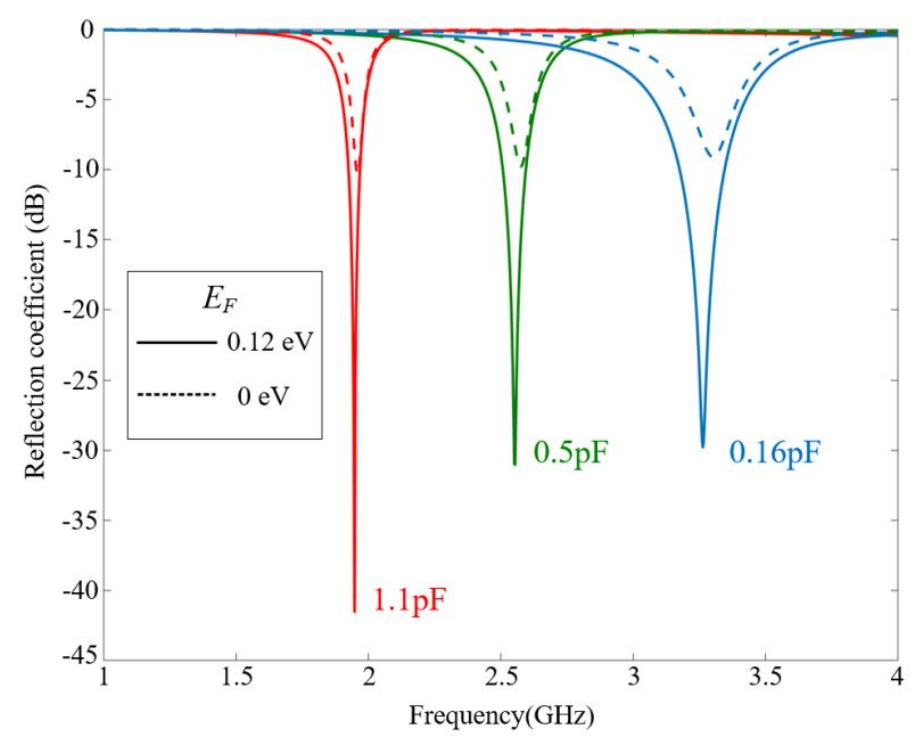

Fig. S1 The simulated reflection coefficient of the proposed metasurface with different capacities and Fermi levels for $y$ polarization. 